Artículos

\title{
EL ESTADO EXPERIMENTADO. AVANCES EN EL ESTUDIO DE LAS RELACIONES ENTRE LAS POLÍTICAS PÚBLICAS DE INCLUSIÓN Y LOS GRUPOS FAMILIARES RURBANOS
}

\author{
Kenbel, Claudia
}

\author{
Claudia Kenbel \\ claudiakenbel@yahoo.com.ar \\ Consejo Nacional de Investigaciones Científicas y \\ Técnicas (CONICET), Universidad Nacional de Río \\ Cuarto (UNRC)., Argentina
}

Intersecciones en Comunicación

Universidad Nacional del Centro de la Provincia de Buenos Aires, Argentina

ISSN: $1515-2332$

ISSN-e: $2250-4184$

Periodicidad: Anual

vol. 1, núm. 15, 2021

intercom@soc.unicen.edu.ar

Recepción: 02 Agosto 2020

Aprobación: 13 Septiembre 2020

URL: http://portal.amelica.org/ameli/

jatsRepo/216/2161348002/index.html

DOI: https://doi.org/10.51385/ic.v1i15.57

Intersecciones en Comunicación sostiene su compromiso con las políticas de Acceso Abierto a la información científica.

Los autores conservan los derechos de autor y conceden a la revista el derecho de la primera publicación de la obra de forma simultánea bajo la Licencia Licencia Creative Commons Atribución-No ComercialCompartir Igual 4.0 Internacional que permite compartir el trabajo con el reconocimiento de su publicación inicial en Intersecciones en Comunicación. Además, se adhiere a la declaración DORA, y su propuesta mejorar la forma de evaluación científica. La revista cuenta con políticas de preservación de documentos digitales a través del Repositorio Institucional Digital de Acceso Abierto de la Universidad Nacional del Centro de la Provincia de Buenos Aires (https:// www.ridaa.unicen.edu.ar) Intersecciones en Comunicación adhiere a las normas internacionales y prácticas respecto a los aspectos éticos que deben seguirse en el proceso de publicación de artículos originales. En este sentido adhiere a lo que sugiere el Committee on Publications Ethics (https://publicationethics.org/resources/ guidelinesnew/principles-transparency-and-best-practice-scholarlypublishing)

\section{c) (i) (2)}

Esta obra está bajo una Licencia Creative Commons AtribuciónNoComercial-CompartirIgual 4.0 Internacional. 


\section{PREOCUPACIONES PREVIAS, DURANTE Y POST PANDEMIA}

Desde hace un tiempo buscamos comprender cómo se produce, reproduce o discute el orden social urbano moderno y las tensiones que se generan cuando se reconocen distintos modos de ser ciudad, y en ese marco, de pensar y planificar la inclusión de sus habitantes y el futuro que se proyecta. Temas que se ponen aún más de relieve en el actual contexto en los siguientes términos:

La pandemia nos igualó; y acto seguido, también nos mostró la profunda desigualdad en la que vivíamos. Por ejemplo, entre
algunos de nosotros parece haber crecido la conciencia del propio privilegio: el de tener una casa habitable, un trabajo, un
sueldo asegurado (o ahorros disponibles), educación, alimentos, salud, seguridad. Otros, posiblemente, nos encontramos
por primera vez compartiendo aquellos problemas que antes sentíamos lejos (no llegar a fin de mes, no poder pagar el
alquiler, subalimentarnos, etc.), aunque "normalmente" sí atravesaban a los sectores más frágiles o vulnerables de nuestra
sociedad (trabajadores informales, desocupados, precarizados, pobres, etc.). Pero, ¿modificó la pandemia nuestro vínculo
con la desigualdad? ¿De qué forma? Porque ante la expectativa concreta de "pagar por el otro" las actitudes varían entre la
disposición a la solidaridad, por un lado, y la afirmación en el individualismo y la policialización en el vínculo con los demás,
por el otro. (Canelo, 2020: 19)

En línea con lo expresado por la autora, decíamos en un trabajo reciente (Kenbel y Corredor, 2020: 11) que en este momento las desigualdades se volvieron más explícitas en el continente más desigual de todos. Desigualdades porque no todos tienen una vivienda en condiciones para realizar el aislamiento, ni necesariamente el acceso al agua potable para la higiene personal y familiar varias veces al día tal como se nos pide desde las comunicaciones oficiales, o una computadora para seguir con las clases en modalidad virtual. En ese sentido, la problemática de fondo tiene como eje de debate principal a la desigualdad en sus múltiples manifestaciones, al rol del estado a través de la implementación (o la omisión) de políticas públicas de inclusión y a las experiencias, expectativas y memorias de grupos familiares rurbanos[1]. Es decir de familias que utilizan carros con caballos en ambientes urbanos como parte de una estrategia histórica de subsistencia. Su presencia en las ciudades pone de manifiesto tensiones de sentidos entre las concepciones sostenidas desde las políticas públicas que buscan su regulación e inclusión, las construcciones mediáticas sobre problemáticas que los vinculan -por ejemplo las referidas al tránsito y el cuidado de los animales- y las experiencias de los actores en su búsqueda por subsistir. En definitiva se habilita la discusión sobre el modelo de ciudad que debe primar, sus derivas y consecuencias para la integración social. Discusión que en el mencionado contexto que atravesamos renueva las preguntas acerca del rol del Estado, las acciones para mitigar las situaciones de desigualdad y cómo se vivencia esta crisis sanitaria en los sectores más vulnerados. Estas son algunas de las motivaciones que nos orientan a profundizar en cómo son las relaciones entre los grupos familiares rurbanos y el Estado en tanto institución reguladora de la vida social y expresión del orden. Vinculaciones que se producen, principalmente, a partir de que el Estado se constituye en ordenador de la sociedad mediante la definición y administración de los derechos y las obligaciones de sus miembros a través de la aplicación de políticas públicas.

En este trabajo nos preguntamos acerca del modo en que se ha abordado esa relación desde diferentes perspectivas teóricas. Cuál ha sido la preocupación central, cómo se ha concebido ese vínculo y qué problemáticas se han enfocado desde cada una de estas miradas. Hemos seleccionado tres posibles, entre muchas otras, a modo de antecedentes de nuestra propia perspectiva. Una proviene de la teoría política que piensa a las relaciones desde las “caras del estado" (O’Donnell, 2010), la segunda lo hace a partir de focalizar en las instituciones y en el rumbo estratégico de las políticas con las implicancias prácticas que esto conlleva sobre todo para los grupos beneficiarios. La tercera proviene de la sociología y se ocupa de las experiencias de los actores en su encuentro con el Estado (Auyero, 2013). Finalmente daremos cuenta del modo en que abordamos esa relación a partir de una incipiente tipología centrada fundamentalmente en la manera en que los grupos rurbanos vivencian su relación con el Estado y las políticas públicas que buscan incluirlos. 


\section{ESTADO Y DESIGUALDAD}

Para comenzar haremos una breve referencia a lo que entendemos por el estado en nuestras coordenadas socio históricas y ligado a determinadas políticas que son las que siembran modos de concebir y sostener expectativas en los diferentes grupos sociales. Es una discusión que nos trasciende, gira en torno a las concepciones políticas pero también a lo que implica la modernidad (Kenbel, 2013) y a las transformaciones que sufrió el mundo con la globalización (Boaventura de Sousa Santos, 2008), lo que fue agregando complejidad a su misión y a sus posibilidades. Brevemente diremos que el estado nos interesa en tanto se lo concibe como el "principal garante y articulador del orden social” (O’Donnell, 2010:111). Junto a la sociedad se constituyen en una unidad, de la cual el estado es la expresión político institucional de aquella, siguiendo a Grassi (2007).

A su vez estas consideraciones se inscriben en el marco del sistema capitalista, desde el cual el rol del estado es, a decir de Grassi (2007: 01), asegurar su “reproducción social” mediante la aplicación de las políticas. En ese sentido nos interesa focalizarnos en las que se denominan "de inclusión social" porque representan la acción consciente del estado por conducir a los sectores sociales más vulnerables dentro de los márgenes del orden vigente. $Y$,en correspondencia, el grupo rurbano que nos interesa presenta necesidades que las requieren. El problema de conocimiento que nos ocupa se ubica así "en la intersección entre las políticas públicas, el análisis de las trayectorias familiares de reproducción y sus memorias grupales y las expectativas frente a la desigualdad y la "inclusión" (Kenbel, 2015: 04).

Como sostuvimos en el inicio, inscribimos la discusión por las políticas públicas en el marco general de la desigualdad en tanto noción relacional, pues a través de ella, dirá Kessler (2016) es posible "reinscribir a la pobreza dentro de la dinámica social y entenderla como un subproducto de las inequidades” (p. 17). También porque conecta la cuestión social con debates políticos y filosóficos de largo aliento, con los principios de justicia que deberían regir en una sociedad, y con las formas de la ciudadanía. Y si hablamos de desigualdad, lo hacemos respecto a su opuesto, es decir la igualdad, y es allí donde, siguiendo a Grassi (2007) se actualiza una tensión consustancial de nuestras sociedades capitalistas: entre "el principio de la igualdad de los individuos (de donde deriva la idea moderna de ciudadanía), y la dependencia operada por la relación salarial” (p. 02). La pregunta de fondo es entonces: ¿Cuál es el rol del estado en esa contradicción entre igualdad/desigualdad? La definición misma de ambos conceptos, en qué esferas, quiénes son los "merecedores de atención y de acciones" (Grassi, 2007: 02) por parte del estado, son ejes controversiales sujetos a posiciones diversas. No obstante, sigue Kessler (2016), resulta necesario articular la mirada multidimensional de la desigualdad con conceptos como exclusión, inclusión, pobreza, bienestar y condiciones de vida en general, "dado que mientras el primero se vincula con procesos sociales más generales, los segundos permiten apreciar más claramente la situación de las poblaciones más vulnerables" (p. 32). Creemos que identificar, nominar, caracterizar a las relaciones que el estado establece con los grupos sociales más desfavorecidos mediante las políticas públicas que buscan la inclusión va en el sentido propuesto, de allí nuestro interés. Veamos a continuación el modo en que las distintas perspectivas que retomamos versan acerca de esa relación.

\section{DESDE LA TEORIA POLITICA EL ESTADO TIENE VARIAS CARAS[2]}

Aquí la preocupación central es el vínculo entre la democracia y el estado. Una democracia que presupone, a decir de O’Donnell (2010) una determinada concepción del ser humano "como un agente que ha logrado, a través de procesos históricos que han variado a lo largo del tiempo y el espacio, el título de ser reconocido, y legalmente respaldado"; es decir en tanto "portador de derechos a la ciudadanía no solo política sino también civil, social y cultural" (p. 9).

Al conceptualizar al estado, O’Donnell (2010) lo hace a partir de cuatro dimensiones: a) como conjunto de burocracias, b) en tanto sistema legal, c) como un intento de foco de identidad colectiva y d) en términos 
de un filtro en relación con su "afuera". No obstante considera que son "insuficientes para su adecuada conceptualización" y es allí cuando propone prestar especial atención a las "diversas caras que ofrece el estado, con especial atención a las que a menudo muestra a los sectores pobres, excluidos y/o discriminados de la población". Concluye en que estos aspectos afectan "directamente la calidad y, en el límite, la existencia misma de la democracia" (p. 18). Las "múltiples caras" constituyen un modo de abordar al estado en tanto "experiencia vivida" (p. 165) por los diversos grupos y clases sociales. O'Donnell (2010) afirma que "el estado está en todos lados y en ninguno". Por ejemplo, la "cara oficial" aparece cuando los gobernantes hablan en actos públicos y en los medios masivos de comunicación, así como cuando edificios, banderas, himnos y desfiles son expuestos en las celebraciones de inauguraciones gubernamentales, en la rememoración del nacimiento o muerte de héroes. Estos eventos, prosigue el autor, ritualizan la unión de los gobernantes con 'su' ciudadanía / nación/pueblo proclamando que estado y gobierno son para el bien de todos. Otra cara "menos visible” está compuesta por la legalidad, la cual cruza nuestras distintas relaciones sociales. Así la describe:

(...) cuando pagamos impuestos o realizamos el examen para conseguir una licencia de conducir-así como, quizás inconscientes de que el estado también está 'ahí' cuando obedecemos una señal de tránsito, vamos a un hospital público, o nuestros hijos asisten a una escuela pública o subsidiada por el estado, y en otras tantas ocasiones, especialmente si vivimos en un entorno urbano y/o modernizado. (...) Estas son las múltiples caras del estado (...) aparece en una variedad apabullante de individuos y cargos; es el 'estado vivido' que ha atraído la atención de perspectivas antropológicas, etnográficas y/o simbólicas. (p. 163)

Estas son a decir del autor, algunas de las múltiples maneras en que se manifiesta a los distintos grupos sociales. Tomando como caso concreto a la justicia dirá O’Donnell (2010) que “en ningún país se administra justicia verdaderamente por igual a los diversos sectores y clases sociales" (p. 170). En tanto los ricos y privilegiados se encuentran con "pocas caras del estado" pues "llevan elaboradas estrategias para eludir la mayor parte de las burocracias estatales", esas pocas veces las caras son frecuentemente "amables" y da algunos ejemplos para graficar. Por caso, viven en complejos habitacionales o edificios de departamentos bien vigilados; cuentan con seguro de salud privado; escuelas y universidades privadas; transporte privado y otros similares. Pero algunas caras son tan amigables, prosigue el autor, y las usan para obtener subsidios, exenciones y otros privilegios que son sumamente difíciles de justificar en términos de equidad social. Además cuando tienen que tratar con la policía o los tribunales, frecuentemente esperan y demandan tratamiento privilegiado.

En cambio la situación para los pobres es totalmente diferente, especialmente para los que viven en regiones rurales marginales y en periferias de grandes ciudades, que pertenecen a enormes y crecientes mercados informales. Las personas viven bajo tal privación que, salvo para individuos y movimientos excepcionales, sobrevivir pasa a ser su abrumadora preocupación; no tienen oportunidades ni recursos materiales, educación, tiempo o incluso energía para hacer mucho más allá de esto. Esto significa que estos individuos son pobres en sentido material, pero las privaciones mencionadas implican que también lo son en sentido legal. La pobreza material y legal es la condición de importantes sectores de la población de los países que albergan regímenes democráticos. Esto lo lleva a calificarlos como pertenecientes a una situación de "ciudadanía de baja intensidad" (p. 171). Sin embargo, en algunas de estas situaciones, incluso con una cara hostil, el estado en cierto sentido todavía está 'ahí', aunque esta presencia es caracterizada por el autor como "evanescente”. Pues puede haber edificios e individuos que invocan poderes legitimados, pero el estado como sistema legal se ha evaporado -o nunca se ha impuesto. El autor concluye que las caras caleidoscópicas del estado son parte de su realidad sociológica vivida, y afirma que es importante que las reconozcamos y reconstruyamos históricamente "en la medida que tienen influencia sobre qué tipo - o calidad- de democracia existe" (p. 183).

\section{LA PERSPECTIVA POLÍTICO INSTITUCIONAL PONE AL ESTADO EN ACCION}

Para proseguir con los aportes desde las ciencias políticas, ahora es el turno de la perspectiva político institucional, del "estado en acción"[3] (Acuña, 2014). Aquí el problema de fondo es la relación entre las instituciones y los procesos sociales. Qué forja qué. Acuña (2014) sostiene que esa pregunta tiene una dimensión abstracta y teórica, a la vez que concreta, la cual está en el “corazón del accionar político cotidiano" y se resume del siguiente modo: "para comprender y resolver nuestros problemas como sociedad y contar con mejores políticas públicas, enos centramos en las instituciones o en otras cuestiones (lo socioeconómico, lo ideológico-cultural, la organización y el comportamiento de los actores, entre otras)?” (p. 13). 
Ahora bien, ¿qué implica un abordaje desde la institucionalidad social? Las instituciones son entendidas, desde esta perspectiva, como "reglas de juego no solo formales y escritas - leyes, reglamentos, manuales operativos-, sino también informales y no escritas" (Repetto, 2014: 22). Son la expresión de la correlación de fuerzas entre aquellos actores con recursos de poder suficientes como para incidir en momentos críticos de la política pública - sea en la definición del problema, sea en la decisión de qué hacer, por citar dos etapas-. La institucionalidad social tiene así relación directa con la calidad en la medida que permite observar el modo en que esa institucionalidad filtrará los problemas sociales: cuales alcanzarán prioridad en la agenda gubernamental y cuáles serán los contenidos de política social que se decidirán, diseñarán e implementarán. Lo cual implica atender a los para qué y a los cómo de las políticas y a sus vínculos con los asuntos críticos de la cuestión social. En los "para qué" de las políticas sociales, una institucionalidad de alta calidad abre la posibilidad de conformar una agenda amplia de problemas a enfrentar, así como el eventual avance hacia aquellas que atiendan a las causas estructurales de los mismos "aprovechando las mejores capacidades de los actores involucrados y las oportunidades que brinda el sistema de reglas formales e informales en 'clave de derechos' “(p. 23). En relación al “' como”, se refiere al modo de dar respuesta a los problemas en un entorno institucional apropiado, mediante una gestión que busque agregar valor y fomentar mejores resultados en tanto los nudos críticos se procesen a partir de la concertación de intereses y transparencia decisional, sin que por ello "desaparezcan los conflictos político-ideológicos" (p. 23) Esta lectura de la institucionalidad se complementa, a decir de Repetto (2014), con un enfoque que atienda a los entornos institucionales de cada sector específico de la política social. No obstante, lejos de ser solo una mirada institucionalista en el sentido de meros formalismos, el factor decisivo lo constituye la política en términos de decidir las acciones a seguir, cuales son las áreas prioritarias, quien tiene derecho a los servicios, quién los proveerá, cómo debe ser distribuido el presupuesto.

Finalmente un interrogante que se abre con esta perspectiva político institucional se refiere al rumbo estratégico de la política social. Lo que implica llamar la atención sobre la necesidad de articulación entre las diversas áreas del estado ya que los problemas sociales que se enfrentan responden a múltiples causas. Pero Repetto (2014) va un paso más y coloca en el debate una pregunta que, para nuestros estudios resulta de importancia, pues podría funcionar a modo de hipótesis en la orientación general de la relación que el estado le propone a los sectores más vulnerables de la sociedad. La pregunta es ¿el país avanza hacia una matriz de política social que coloca en primer plano al ciudadano o al trabajador? La tensión no es solo conceptual, sino “práctica al extremo" en los siguientes términos:

...por ejemplo en términos de cómo se financia la política social (¿con impuestos generales?, ¿con aportes de los actores del mundo del trabajo?) en la clásica tensión de cómo combinar universalismo con acciones focalizadas. Esto tiene implicancias prácticas en el tipo de coalición sociopolítica que se requiere para consolidar los avances logrados y al mismo tiempo, profundizar la perspectiva de ampliación de derechos no solo en el plano formal, sino en sus efectividades prácticas" (Repetto, 2014: 67)

\section{ENCUENTROS INTIMOS DESDE LAS EXPERIENCIAS DE LOS ACTORES}

En tanto las perspectivas anteriores pusieron el foco en la teoría política y las instituciones, esta postura se centra en la relación entre ciudadanía, democracia y desigualdad como "rasgo definitorio del carácter real de la ciudadanía y del funcionamiento de la democracia" (Auyero, 2013: 39). Particularmente en "Pacientes del estado", texto que aquí retomamos, el foco empírico son las "prácticas relacionales que vinculan el funcionamiento cotidiano del Estado con la vida de los subordinados” (p. 20).

Auyero (2013) argumenta acerca de la centralidad del Estado en la vida de los sectores más vulnerables, a pesar del contexto de globalización económica y de hegemonía neoliberal. Sostiene que continúa siendo un actor clave porque "cuando se trata de los pobres, está, en palabras de Akhil Gupta (1995: 375), profundamente implicado hasta en el más mínimo aspecto de la vida cotidiana” (Auyero, 2013: 20). Dado 
que los encuentros cotidianos con las burocracias estatales le dan "forma y marco concreto a lo que sería de otra forma una abstracción ('el Estado'), estos encuentros son fundamentales en la construcción cotidiana del Estado" (p. 20).

Entendemos que es posible vincular el razonamiento de Auyero con las "caras del estado" que propone O’Donnell (2010). Pues sostiene que los estados "declaran” con palabras, señales y recursos" y lo hacen "a través de relaciones sociales concretas y del establecimiento de rutinas, rituales e instituciones que funcionan en nosotros". En lugar de ser solo un "aparato burocrático más o menos funcional, el Estado es además un poderoso lugar de producción cultural y simbólica” (p. 20). De allí que el autor sostiene que su libro se suma al llamado de ir hacia un análisis relacional de los procesos enfocados sobre la interacción cotidiana del Estado con los sectores urbanos pobres. Auyero menciona que estas preocupaciones tienen su trayectoria en el ámbito de las ciencias sociales y ponen el énfasis en la reconstrucción del Estado a través de las prácticas cotidianas de la gente. La atención está puesta tanto en las formas institucionales, las estructuras organizativas y las capacidades, -lo cual se identifica con la perspectiva político institucional mencionada anteriormente-, así como en lo que el estado significa para los actores. Estos significados, a decir del autor, se constituyen a partir de "archivos, órdenes, memos, estadísticas, informes, peticiones, inauguraciones, inspecciones, transferencias, la cotidianeidad de los intercambios entre las burocracias y los burócratas y los ciudadanos" (p.21). Este último grupo de preocupaciones centradas entonces en lo que el estado significa para los distintos grupos sociales continúa notablemente poco estudiado en contraste con otras investigaciones dedicadas a las maquinaciones de los líderes del Estado, los cambios en las grandes políticas. Finalmente concluye Auyero (2013)-, el Estado es entendido como una estructura abstracta a nivel macro, a la vez que un conjunto de instituciones a nivel micro con las cuales los habitantes urbanos pobres interactúan de manera directa e inmediata.

Estas relaciones son analizadas por el autor y su equipo de investigación a través del encuentro entre los empleados públicos y los ciudadanos individuales al momento de tramitar distintas acciones estatales con el propósito de mostrar cómo en esas interacciones los primeros dan "lecciones políticas" y moldean las expectativas de los beneficiarios: El foco empírico está en la interacción cotidiana con el Estado local, si bien reconoce que la ciudadanía es una cuestión multiescalar, cuestión con la que coincidimos.

\section{LAS EXPERIENCIAS RURBANAS CON EL ESTADO: RELACIONES Y EXPECTATIVAS DE INCLUSION RECAPITULACIONES}

Antes de avanzar en este apartado vamos a recapitular algunos aspectos centrales de las perspectivas presentadas que ofician como escenario para nuestro modo de comprender el problema de conocimiento.

Acordamos en concebir al Estado como una unidad compleja junto a la sociedad, lo que significa que es "simultáneamente medio y expresión de constitución de la sociedad", dicho de otro modo, "la sociedad es inmediatamente un orden político cuya expresión institucional es el Estado” (Grassi, 2007: 01). Esta concepción a su vez no lo es en el abstracto, sino que nos referimos a un Estado que se manifiesta en un marco sociopolítico con determinadas características, concretamente en el sistema capitalista. Lo que tiene como efecto inmediato entender que una de sus atribuciones principales es la de articular y garantizar el orden social que busca establecerse y legitimarse. Por lo que si en un nivel general nuestras preocupaciones son por el orden social urbano moderno en el marco del sistema capitalista y desde América Latina (Kenbel, 2013, 2020), en un plano concreto una de sus manifestaciones será el Estado, más precisamente a través de las diversas políticas públicas que se implementan para organizar a la sociedad. Cómo esas políticas se argumentan, sostienen y aplican se vincula al modo en que el orden es concebido y reproducido.

Acordamos también en remarcar su centralidad para los distintos grupos sociales, fundamentalmente para los sectores en situación de vulnerabilidad hasta en el más mínimo aspecto de su vida cotidiana, parafraseando a Auyero (2013). Esa presencia se manifiesta a través de distintas “caras" de acuerdo a los sectores a los cuales 
se dirige. El rostro que le muestra a los grupos que nos interesa es caracterizado como evanescente, incluso hostil (O’Donnell, 2010), pero aun así el estado 'está ahí'. Otro aspecto complementario a esa presencia errática del Estado, lo aporta la perspectiva político institucional, justamente al poner el acento en el rumbo estratégico de las acciones estatales, es decir en su "matriz política". Dicho de otro modo, cuales problemas sociales tendrán prioridad en la agenda gubernamental, los fundamentos y los contenidos de las acciones para revertirlos, así como el horizonte último de tales políticas. Lo cual, parafraseando a Repetto (2014), tiene consecuencias prácticas tales como decidir de qué manera se financian las políticas, si son aplicaciones universales o focalizadas, de largo o corto plazo, y cuales los consensos necesarios para consolidar tales caminos, así también para profundizar la perspectiva de ampliación de derechos ciudadanos.

En cuanto al tipo de abordaje que proponen las perspectivas mencionadas, podríamos concluir que mientras las dos primeras se centran fundamentalmente en el Estado en cuanto organizador de la vida social, la tercera - desde la sociología- lo hace desde las experiencias de los grupos sociales más vulnerados. En términos del "estado vivido" (O’Donnell, 2010), experimentado y significado (Auyero, 2013) se lo entiende como un "poderoso lugar de producción cultural y simbólica” (Auyero, 2013: 20), marco a partir del cual es posible que se configuren expectativas, sentires, frustraciones y posibilidades.

Con estos acuerdos avanzamos entonces al modo en que comprendemos la relación entre los grupos familiares rurbanos y el estado.

\section{Mirar DESDE LA COMUNICACIÓN}

Es importante comenzar este apartado atendiendo a la manera en que entendemos a la comunicación. En instancias anteriores buscamos comprender la circulación de ciertas concepciones hegemónicas y alternas del orden social urbano moderno a partir de reconocer tensiones de sentido[4] (Kenbel, 2013:51). Este abordaje implicó considerar no sólo la problemática de los medios de comunicación y su rol de "agendadores" de la realidad o "constructores" de las versiones creíbles, sino también a las mediaciones históricas y simbólicas que los diversos sectores sociales ponen en juego a la hora de configurarse como emisores o receptores válidos. Perspectiva que abre interrogantes atentos tanto a las "estructuras y dinámicas de poder, pero también abierta a interpretar las rupturas y procesos emergentes" (Cimadevilla, 2007). Nos ubicamos en la línea de pensamiento latinoamericano de los investigadores de la comunicación que apunta a "trascender el aspecto estrictamente técnico y del desarrollo de habilidades, para ubicarla sobre todo en el espacio de las relaciones entre sujetos, enmarcados en contextos sociales y culturales" (Uranga, 2016: 30). Una perspectiva entonces que atiende a los modos de percibir y significar la vida cotidiana, desde la experiencia propia, en diálogo con las diferencias y los conflictos.

Este breve repaso por las preocupaciones de entonces son el antecedente de nuestro interés por las relaciones entre el Estado y los grupos familiares rurbanos a través de reconocer, caracterizar y profundizar en las experiencias que estos tienen en torno a la aplicación de políticas públicas que buscan su inclusión.

Hasta el momento de esta presentación, hemos avanzado del siguiente modo. En relación a la identificación de políticas como uno de los primeros pasos para establecer los términos de la relación con los grupos rurbanos, aprendimos que no se trata de buscarlas "a priori", sino de avanzar a partir de la experiencia acumulada con el grupo de interés. De este modo identificamos ámbitos de aplicación de las políticas. Por caso, en torno a la promoción social - tarifas sociales para la provisión de servicios públicos como la energía eléctrica domiciliaria, pasando por las AUH, no contributivas -, la salud -acceso a medicamentos, tratamientos propios y de familiares, situaciones de discapacidad-, el trabajo -acciones en torno a la recuperación de residuos y el uso de la tracción a sangre. Estas acciones estatales, que a partir de lo descripto, se constituyen en las caras que el Estado, sobre todo local, le muestra a los grupos rurbanos. A su vez tales políticas pueden estar encaminadas de modo individual -por ejemplo, incentivos al salario por cada trabajador-, por grupo familiar o ser de índole organizacional-por caso, apoyo a infraestructura para 
emprendimientos autogestionados-. Algunas acciones son para el conjunto de las poblaciones en situación de desigualdad, en tanto otras son focalizadas, por ejemplo, para quienes se dedican a ciertas actividades.

Identificadas las acciones, lo cual en el contexto de pandemia y post pandemia, demandará actualizarlas a la luz de los acontecimientos, y considerando las experiencias, las memorias y las expectativas de los grupos familiares rurbanos, recogidas en distintas instancias de investigación y acción[5], es posible avanzar en una incipiente tipología. Hemos esbozado la consideración de dos planos, a) uno referido al condicionamiento en la relación con el estado según las percepciones de los grupos familiares rurbanos. Y otro b) alrededor de la satisfacción/insatisfacción de sus necesidades.

Si nos ubicamos en los condicionamientos, estos pueden dar lugar a relaciones que hemos dado en llamar "disruptivas" o "aceptables". Resultan disruptivas cuando en la relación que el estado establece se pone en juego de algún modo la sobrevivencia del grupo familiar. Por ejemplo, ante una política que ofrece el reemplazo de la tracción a sangre por una motocarga, ceder los animales es parte y condición para la obtención del vehículo. En este caso el grupo familiar rurbano se ve en la situación de optar por un tipo de vehículo u otro, poniendo en riesgo el sistema de movilidad tradicional que utiliza para trabajar. A partir de los relatos de los grupos familiares es posible advertir que este cambio genera incertidumbre respecto a lo que sucede si el vehículo se rompe, o qué sucede si no se logra reunir el dinero para la carga de combustible, una rotura o el pago de una cuota por el vehículo. Para seguir con el ejemplo, durante el proceso que dura la relación con el estado para la obtención del vehículo se producen fricciones, desencuentros y discusiones entre los beneficiarios y el personal a cargo de las acciones. Una relación disruptiva se puede identificar a partir de las frustraciones y los desgastes que los actores mencionan en los distintos encuentros que mantienen con el estado.

En cambio un condicionamiento se percibe como "aceptable" si la relación que se establece no implica poner en riesgo la sobrevivencia del grupo, sino que se lo acepta como parte de la política implementada. Por caso, cuando se ofrecen ayudas económicas en forma de becas de trabajo, las cuales suponen una contraprestación percibida como parte de aquello que se otorga. Estas relaciones que no ponen en riesgo la sobrevivencia del grupo crean un marco propicio para generar expectativas de cambio, crecimiento o mejoras en los actores.

A su vez si nos ubicamos en el plano de la satisfacción/insatisfacción que perciben los grupos familiares al establecer relaciones con el estado, nos encontramos con relaciones que podríamos llamar "satisfactorias" e "insatisfactorias". Nos referimos a una relación como satisfactoria cuando los grupos rurbanos perciben que han logrado su cometido, por ejemplo, un trámite que comienza y termina en un período de tiempo razonable para su vida cotidiana. Que puede ser obtener ayuda económica, tramitar productos alimenticios, solicitar un turno médico o en ANSES, así como beneficios para el trabajo (vehículo, elementos de seguridad, becas). Las expectativas de los grupos familiares son óptimas. Por el contrario resulta insatisfactoria la relación cuando los grupos familiares rurbanos perciben que no han logrado el cometido por el cual se relacionan con el estado. Por caso un trámite que inicia con una gestión y luego se "cae", cuando se cortan los fondos destinados a tal ayuda o bien cuando la ayuda no era la esperada.

Los planos - del condicionamiento y de la satisfacción- están en interacción constante, de modo tal que una relación de condicionamiento disruptivo puede ser percibida como satisfactoria o insatisfactoria, según el objetivo que se busque resulte en un logro o no. Lo mismo, una relación aceptable puede constituirse en satisfactoria o no de acuerdo al beneficio que se trate. Por último, estos planos que apenas son un esbozo en un proceso de investigación que continúa, tienen como marco general la "matriz política" de las políticas públicas, es decir, el horizonte último que se dirime socialmente en torno a la inclusión de los grupos sociales más vulnerables en el orden vigente. 


\section{APENAS UNAS CONCLUSIONES}

Como mencionamos en el inicio, una serie de preocupaciones relacionadas a la problemática de la desigualdad, al rol del Estado y a la experiencia de grupos familiares rurbanos, se actualizan en este contexto de pandemia y post pandemia. “¿Modificó la pandemia nuestro vínculo con la desigualdad? ¿De qué forma?” (Canelo, 2020), decíamos en el inicio. Qué sucedió con el cotidiano de los grupos familiares rurbanos, con las medidas de aislamiento y distanciamiento social, cómo afrontaron y afrontan la crisis no solo sanitaria sino social y económica en un contexto de creciente pobreza, no solo para Argentina, sino con repercusiones en el modelo económico global. Es decir, si bien el caso de los grupos sociales que han sido parte de nuestras investigaciones presenta características de múltiples vulnerabilidades estructurales, será materia de investigación y comprensión por estos días, el modo concreto en que las medidas asociadas a la pandemia repercutieron en sus estrategias de vida. En ese sentido, nuestros procesos de investigación también se verán afectados. Así ha sido incluso en el marco de este trabajo, pues las preguntas que teníamos se actualizan con nuevos ribetes: ¿Cuáles son los rumbos estratégicos que se están pensando para la sociedad argentina post pandemia? ¿Desde qué matrices se están pensando las medidas asociadas al, por ejemplo, Ingreso Familiar de Emergencia (IFE) o al Salario Universal en debate? ¿Qué pasa con el sistema de salud? ¿Y con la educación? Al haber pasado varios meses de actividad concomitante o nula en varios rubros de la economía, ¿qué repercusiones tiene para el conjunto social? ¿Y para aquellos que venían viviendo privaciones anteriores como las familias rurbanas? ¿Qué sucederá con el sector informal y su reconocimiento? ¿Cuál es en definitiva el rol del estado en este tiempo que vivimos? ¿Cómo vive esta situación un grupo familiar con escasos márgenes de maniobra si no es posible por caso salir a buscar una changa? ¿Cómo se reconfiguran las organizaciones territoriales y las demandas a atender? Creemos que el camino iniciado en el vínculo estado y grupos familiares rurbanos a través de las políticas públicas que buscan su inclusión debe prestar atención a los nuevos requerimientos, combinando historicidad, micro relatos, sentires, expectativas, frustraciones y sobre todo, salidas junto a los actores involucrados.

\section{REFERENCIAS}

ACUÑA, C (compilador) 2014 El estado en acción. Fortalezas y debilidades de las políticas sociales en la Argentina. Primera Edición. Págs. 13-17. Buenos Aires. Siglo XXI Editores.

AUYERO, J 2013 Pacientes del estado. Primera Edición. Buenos Aires, EUDEBA.

CANELO, P 2020 Igualdad, solidaridad y nueva estatalidad. El futuro después de la pandemia En El futuro después del COVID-19 Comp. Alejandro Grimson. Programa Argentina Futura. Págs. 17- 25. Ciudad de Buenos Aires, Argentina. Disponible en https://www.argentina.gob.ar.

CIMADEVILLA, G 2007 “Contrapuntos con Lefebvre. De la revolución urbana a la rurbanidad. Ponencia VI Bienal Iberoamericana deComunicación. Córdoba. UNC.

DE SOUSA SANTOS, B 2008 Pensar el estado y la sociedad: desafíos actuales. La Paz (Bolivia). Muela del diablo Editores, COMUNA y CLACSO.

GRASSI, E 2007 La política social, las necesidades sociales y el principio de la igualdad: tendencias y perspectivas en el contexto ¿post neoliberal? Panel en el Congreso Latinoamericano y Caribeño de Ciencias Sociales. Quito (Ecuador). FLACSO SENPLADES.

KENBEL, C 2013 Circuitos culturales y tensiones de sentidos. La rurbanidad según las memorias sociales en la ciudad de Río Cuarto. Tesis doctoral inédita. Facultad de Ciencia Política y Relaciones Internacionales. Universidad Nacional de Rosario.

KENBEL, C 2015 "Las políticas públicas en la perspectiva de la rurbanidad: Familias, memorias y expectativas de “inclusión”, Plan de Trabajo Ingreso a Carrera de Investigadora, Consejo Nacional de Investigaciones Científicas y Técnicas. 
KENBEL, C y GARCIA CORREDOR, C 2020 Comunicación para imaginar futuros deseables para América Latina En Revista Contratexto n 33. Págs. 11-18. Lima, Perú. Disponible en https://revistas.ulima.edu.pe.

KENBEL, C, DEMARCHI, P, GALIMBERTIS 2020 Iconos de la rurbanidad. Actores, prensa, tecnologías y políticas de reordenamiento rurbano en tiempos modernos. Págs. 8-25; 31- 122. Río Cuarto, Argentina. Disponible en: https://www.unirioeditora.com.ar.

KESSLER, G 2016 Controversias sobre la desigualdad. Argentina 2003-2013. Pàgs. 13-55. Ciudad de Buenos Aires, FCE.

O’DONNELL, G 2010 Democracia, agencia y estado. Teoría con intención comparativa. Primera Edición. Buenos Aires, Prometeo Libros.

REPETTO, F 2014 Políticas sociales: una mirada político institucional a sus reformas, desafíos e impactos En El estado en acción. Fortalezas y debilidades de las políticas sociales en la Argentina. Carlos Acuña (compilador). Primera Edición. Págs. 19-69. Buenos Aires. Siglo XXI Editores.

URANGA, W 2016 Conocer, transformar, comunicar. Primera Edición. Págs. 27-58. Buenos Aires. Editora Patria Grande.

\section{Notas}

[1] El concepto de rurbanidad, retoma una vieja preocupación expresada por Le Play en el siglo XIX y por Anderson o Guigou en los años ' 60 del siglo XX respecto de la tendencia a la "extinción de lo rural" y la total "artificialización del ambiente". Aunque el concepto fue particularmente propuesto por Charles Galpin a inicios de ese siglo (1918) en los Estados Unidos para identificar un movimiento de reforma social preocupado por las transformaciones industriales y el destino de los ambientes y actores rurales. Una versión actualizada del concepto y sus implicancias para los estudios que desarrollamos se encuentra en "Iconos de la rurbanidad. Actores, prensa, tecnología y políticas de reordenamiento rurbano en tiempos modernos” (Kenbel, Demarchi y Galimberti, 2020, UNIRIO Editora)

2 Este apartado es una reescritura del trabajo "El estado experimentado. Un enfoque en construcción", presentado en el marco del Encuentro Nacional de Carreras de Comunicación del año 2016. Publicado y disponible en el libro de actas del mencionado evento ENACOM 2016.

3 Hacemos alusión al título del libro compilado por Carlos Acuña: "El estado en acción. Fortalezas y debilidades de las políticas sociales en la Argentina”. 2014. Siglo XXI Editores, Fundación OSDE.

4 Llamamos tensiones de sentido a las discordancias conceptuales provenientes de los cruces de lecturas hegemónicas y alternasen relación a la legitimidad del orden social establecido. En el caso de la tesis doctoral, tales tensiones de sentido, aparecieron materializadas en las memorias sociales en torno a la aplicación de políticas públicas (hitos conflictuantes) con incidencia en el grupo social rurbano. Algunas de las tensiones manifiestas en relación al orden social urbano moderno se refieren a a) la socialidad, b) a las actividades económicas, c) la vida política-institucional y d) la cultura y la asignación de sentidos. (Kenbel, 2013: 51, 52, 53 y 54)

5 Nos referimos a proyectos de investigación financiados y evaluados por la Secretaría de Ciencia y técnica de la Universidad Nacional de Río Cuarto de manera ininterrumpida desde el año 2003, así como a proyectos de extensión que han tenido como protagonistas a las familias rurbanas (Secretaría de Políticas Universitarias, 2015). Y más recientemente a un PIODO (Proyecto de Investigación Orientado a la Demanda y la Oferta) financiado por el MINCYT Córdoba y relacionado a la construcción de tecnologías sociales para fortalecer procesos asociativos de recuperadores de residuos (2017-2018). 